\title{
Structural study on mutant $\alpha$-L-iduronidases: insight into mucopolysaccharidosis type I
}

\author{
Kanako Sugawara $\cdot$ Seiji Saito $\cdot$ Kazuki Ohno $\cdot$ \\ Torayuki Okuyama $\cdot$ Hitoshi Sakuraba
}

Received: 16 January 2008/ Accepted: 15 February 2008/Published online: 14 March 2008

(C) The Japan Society of Human Genetics and Springer 2008

\begin{abstract}
To elucidate the basis of mucopolysaccharidosis type I (MPS I), we constructed structural models of mutant $\alpha$-L-iduronidases (IDUAs) resulting from 33 amino acid substitutions that lead to MPS I (17 severe, eight intermediate, and eight attenuated). Then, we examined the structural changes in the enzyme protein by calculating the number of atoms affected and determined the root-meansquare distance (RMSD) and the solvent-accessible surface area (ASA). In the severe MPS I group, the number of atoms influenced by a mutation and the average RMSD
\end{abstract}

Disclaimers: None

Electronic supplementary material The online version of this article (doi:10.1007/s10038-008-0272-4) contains supplementary material, which is available to authorized users.

K. Sugawara $\cdot$ H. Sakuraba $(\bowtie)$

Department of Analytical Biochemistry,

Meiji Pharmaceutical University, 2-522-1 Noshio,

Kiyose, Tokyo 204-8588, Japan

e-mail: sakuraba@my-pharm.ac.jp

S. Saito

Graduate School of Agricultural and Life Science,

The University of Tokyo, Tokyo, Japan

K. Ohno

Center for Biological Resources and Informatics,

Tokyo Institute of Technology, Yokohama, Japan

T. Okuyama

Department of Clinical Laboratory Medicine,

National Center for Child Health and Development,

Tokyo, Japan

Present Address:

K. Ohno

Drug Discovery Research, Astellas Pharm Inc,

Tsukuba, Japan value were larger than those in the attenuated group, and the residues associated with the mutations identified in the severe group tended to be less solvent accessible than those in the attenuated group. The clinically intermediate phenotype group exhibited intermediate values for the numbers of atoms affected, RMSD, and ASA between those in the severe group and those in the attenuated group. The results indicated that large structural changes had occurred in the core region in the severe MPS I group and small ones on the molecular surface in the attenuated MPS I group. Color imaging revealed the distributions and degrees of the structural changes caused by representative mutations for MPS I. Thus, structural analysis is useful for elucidating the basis of MPS I. As there was a difference in IDUA structural change between the severe MPS I group and the attenuated one, except for a couple of mutations, structural analysis can help predict the clinical outcome of the disease.

Keywords Mucopolysaccharidosis type I ·

Hurler syndrome - Scheie syndrome .

Hurler/Scheie syndrome $\cdot \alpha$-L-Iduronidase $~$

Protein structure

\section{Introduction}

Alpha-L-iduronidase (IDUA; E.C.3.2.1.76) is an acid glycosidase involved in the degradation of glycosaminoglycans (GAGs) heparan sulfate and dermatan sulfate. Defects in the IDUA gene result in a human metabolic disease, mucopolysaccharidosis type I (MPS I; McKusick 25280), causing impaired degradation of GAGs and their systemic deposition (Neufeld and Muenzer 2001). The disease exhibits a wide clinical spectrum. The most severe 
phenotype with early onset (Hurler syndrome, MPS IH) involves mental retardation, skeletal deformities, stiff joints, hepatosplenomegaly, corneal clouding, and a shortened life expectancy. The attenuated phenotype with late onset (Scheie syndrome, MPS IS) involves mild skeletal deformities, stiff joints, corneal clouding, and a long life span without mental retardation. Furthermore, there is a clinically intermediate phenotype (Hurler/Scheie syndrome, MPS IH/S). As there are marked differences between the severe and attenuated phenotypes, they must be due to different recessive mutations of the IDUA gene and different expressed products, but the details have not yet been determined.

The IDUA gene spans $19 \mathrm{~kb}$, includes 14 exons (Scott et al. 1992), and is localized to $4 \mathrm{p} 16.3$ (Scott et al. 1990). It encodes a precursor IDUA composed of a 653-amino acid polypeptide, the enzyme being glycosylated and then processed to the mature form (Tylor et al. 1991; Brooks 1993). As to the active site of IDUA, Brooks et al. (2001) predicted the putative acid/base catalyst and nucleophile residues to be E182 and E299, respectively. Then, they expressed site-directed mutants E182A and E299A in Chinese hamster ovary (CHO)-K1 cells and showed that the mutant proteins were catalytically inactive. These results suggest that these residues are important for the catalytic mechanism of IDUA.

So far, many gene mutations causing MPS I have been reported (Neufeld and Muenzer 2001; Yamagishi et al. 1996). Among them, gross alterations of the IDUA gene have been generally found in patients with the severe form, but missense mutations comprising the majority of mutations in MPS I have been identified in both the severe and milder forms, i.e., the intermediate and attenuated ones. As a recombinant human IDUA (laronidase; Genzyme, MA, USA) has been developed and introduced to enzyme replacement therapy for MPS I (Brooks 2002), prediction of the clinical outcome of the disease is becoming more and more important for determining the proper therapeutic schedule in the early stage of MPS I. To elucidate the basis of MPS I and to predict the clinical outcome of this disease, structural information on a defective IDUA is very important. However, only few reports on this issue have been published. Rempel et al. (2005) constructed a homology model for IDUA based on the crystal structure of the $\beta$-xylosidase from Thermoanaerobacterium saccharolyticum, predicted the location of residues associated with MPS I in the IDUA structure, and analyzed the structural differences in amino acid chains.

In this study, we built structural models of mutant IDUA proteins resulting from amino acid substitutions responsible for severe, intermediate, and attenuated MPS I. We then examined their structural changes by calculating the number of atoms influenced by the amino acid substitutions and by determining the root-mean-square distance (RMSD) and the solvent-accessible surface area (ASA). Furthermore, we examined the distributions and degrees of threedimensional structural changes caused by representative mutations by means of color imaging.

\section{Materials and methods}

Amino acid substitutions causing severe, intermediate, and attenuated MPS I

So far, more than 40 missense mutations associated with MPS I have been reported. We here analyzed 33 amino acid replacements for which the phenotypes have been clearly described (17 severe, eight intermediate, and eight attenuated). The amino acid substitutions, their phenotypes, and references are summarized in Table 1.

Structural modeling of mutant IDUA proteins, and calculation of the number of atoms influenced by amino acid substitutions responsible for MPS I

Structural models of mutant IDUAs were built using molecular modeling software TINKER, developed by Ponder et al. (Department of Biochemistry and Molecular Biophysics, Washington University) (Kundrot et al. 1991; Dudek and Ponder 1995; Kong and Ponder 1997; Pappu et al. 1998; Ren and Ponder 2003). As a template, the homology model of human IDUA (PDB: 1Y24) (Rempel et al. 2005) was used, and energy minimization was performed. The root-mean-square graduate value was set at $0.05 \mathrm{kcal} / \mathrm{mol} \AA$. Then, each mutant model was superimposed on the wild-type structure based on the $\mathrm{C} \alpha$ atoms by the least-square-mean fitting method (Kabsch 1976, 1978; Sakuraba et al. 2000, 2004; Matsuzawa et al. 2003, 2005). We defined that the structure was influenced by an amino acid substitution when the position of an atom in a mutant differed from that in the wild type by more than the cutoff distance $(0.15 \AA)$ based on the total RMSD, as described previously (Matsuzawa et al. 2005). We calculated the numbers of influenced atoms in the main chain and the side chain.

Determination of RMSD values of all atoms in mutant IDUAs

To compare the influences on conformational changes in IDUA by amino acid substitutions, the RMSD values of all atoms in mutant IDUAs were calculated according to the standard method (Weiner et al. 1984), and the average RMSDs for the severe, intermediate, and attenuated MPS I groups were determined. 
Table 1 Mucopolysaccharidosis type I (MPS I) mutations, structural changes in $\alpha$-L-iduronidases (IDUA), and phenotypes

\begin{tabular}{|c|c|c|c|c|c|c|}
\hline \multirow[t]{2}{*}{ Mutation } & \multicolumn{2}{|c|}{ Numbers of affected atoms } & \multirow[t]{2}{*}{$\operatorname{RMSD}(\AA)$} & \multirow[t]{2}{*}{$\operatorname{ASA}\left(\AA^{2}\right)$} & \multirow[t]{2}{*}{ Phenotype } & \multirow[t]{2}{*}{ Reference } \\
\hline & Main chain & Side chain & & & & \\
\hline G51D & 142 & 190 & 0.155 & 0 & Severe & Venturi et al. (2002) \\
\hline A75T & 0 & 0 & 0.002 & 40.7 & Severe & Clarke et al. (1994) \\
\hline A160D & 76 & 80 & 0.067 & 6.1 & Severe & Venturi et al. (2002) \\
\hline E182K & 227 & 277 & 0.139 & 17.3 & Severe & Matte et al. (2003) \\
\hline P183R & 223 & 256 & 0.14 & 0.4 & Severe & Venturi et al. (2002) \\
\hline G208D & 57 & 62 & 0.066 & 5.2 & Severe & Matte et al. (2003) \\
\hline G208V & 103 & 88 & 0.091 & 5.2 & Severe & Beesley et al. (2001) \\
\hline L218P & 35 & 48 & 0.059 & 9.5 & Severe & Bunge et al. (1994a) \\
\hline I270S & 0 & 3 & 0.003 & 60.1 & Severe & Laradi et al. (2005) \\
\hline D315Y & 91 & 90 & 0.124 & 17.3 & Severe & Li et al. (2002) \\
\hline A327P & 3 & 4 & 0.019 & 0.3 & Severe & Bunge et al. (1994a) \\
\hline D349Y & 84 & 119 & 0.095 & 3.2 & Severe & Matte et al. (2003) \\
\hline $\mathrm{R} 363 \mathrm{C}$ & 47 & 48 & 0.041 & 52.7 & Severe & Yogalingam et al. (2004) \\
\hline Т366P & 64 & 56 & 0.081 & 12.6 & Severe & Scott et al. (1995) \\
\hline T388R & 68 & 74 & 0.056 & 14.3 & Severe & Rempel et al. (2005) \\
\hline R489P & 95 & 113 & 0.093 & 27.7 & Severe & Bunge et al. (1994b) \\
\hline P496R & 533 & 511 & 0.245 & 0 & Severe & Beesley et al. (2001) \\
\hline A79V & 11 & 6 & 0.025 & 53.7 & Intermediate & Yogalingam et al. (2004) \\
\hline $\mathrm{H} 82 \mathrm{P}$ & 125 & 159 & 0.127 & 47.2 & Intermediate & Clarke et al. (1994) \\
\hline E178K & 155 & 234 & 0.111 & 3.6 & Intermediate & Venturi et al. (2002) \\
\hline S260F & 0 & 2 & 0.011 & 64.2 & Intermediate & Matte et al. (2003) \\
\hline L346R & 66 & 91 & 0.055 & 14.8 & Intermediate & Teng et al. (2000) \\
\hline T364M & 0 & 0 & 0.002 & 39.2 & Intermediate & Lee-Chen and Wang (1997) \\
\hline L490P & 28 & 24 & 0.056 & 111.4 & Intermediate & Tieu et al. (1995) \\
\hline P496L & 135 & 121 & 0.115 & 0 & Intermediate & Tieu et al. (1995) \\
\hline R89Q & 109 & 144 & 0.077 & 0.8 & Attenuated & Scott et al. (1993) \\
\hline C205Y & 28 & 38 & 0.05 & 1.3 & Attenuated & Beesley et al. (2001) \\
\hline H240R & 5 & 0 & 0.011 & 146.6 & Attenuated & Beesley et al. (2001) \\
\hline A319V & 0 & 0 & 0.002 & 48.8 & Attenuated & Beesley et al. (2001) \\
\hline N350I & 6 & 15 & 0.019 & 57.5 & Attenuated & Matte et al. (2003) \\
\hline Q380R & 17 & 22 & 0.031 & 26.9 & Attenuated & Beesley et al. (2001) \\
\hline $\mathrm{R} 383 \mathrm{H}$ & 21 & 29 & 0.031 & 41.9 & Attenuated & Clarke et al. (1994) \\
\hline R492P & 14 & 13 & 0.022 & 145.6 & Attenuated & Tieu et al. (1995) \\
\hline
\end{tabular}

$R M S D$ root-mean-squares distance, $A S A$ solvent-accessible surface area

Determination of ASA of amino acid residues

The ASA of each amino acid residue in the wild-type IDUA was calculated using ACCESS (McDonald and Thornton 1994). As an input structure, the homology model of human IDUA was used. Then, the average ASA values of the residues for which a substitution had been identified in the severe, intermediate, and attenuated MPS I groups were determined.

Statistical analysis

Statistical analysis to determine the differences in the numbers of influenced atoms, the RMSD, and the ASA between the severe MPS I and attenuated groups, between the severe MPS I and milder ones (the intermediate and attenuated groups), between the severe MPS I and intermediate ones, and between the intermediate MPS I and attenuated ones was performed by means of the $F$ test, and then Welch's $t$ test, it being taken that there was a significant difference if $P<0.05$.

Color imaging of the atoms influenced by representative amino acid substitutions causing MPS I

Color imaging of the influenced atoms in the threedimensional structure of IDUA was performed for amino 
acid substitutions including P496R (severe), H240R (attenuated), and E182K (severe). For these cases, clinical and biochemical investigations had been performed in detail (Brooks et al. 2001; Beesley et al. 2001; Matte et al. 2003). The analysis was based on the distances between the wild type and mutant to determine the influence of the amino acid substitutions geographically and semiquantitatively according to the method previously described (Matsuzawa et al. 2005).

\section{Results}

Numbers of atoms influenced by amino acid substitutions associated with MPS I

According to the structural model of human IDUA, the IDUA consists of two domains: a $(\beta / \alpha)_{8}$-barrel domain, and an antiparallel $\beta$-sheet domain (Rampel et al. 2005). To elucidate the basis of MPS I and to determine the relationship between the structural change caused by an amino acid substitution and the MPS I phenotype, we identified the locations of residues for amino acid substitutions and those of the putative acid/base catalyst (E182) and nucleophile (E299) in the structure of the wild-type IDUA (Fig. 1).

Then, we built structural models of the mutant IDUAs and calculated the numbers of atoms influenced by the amino acid substitution for each mutant model (Table 1). For the severe MPS I group, the averages for the influenced atoms in the main chain and the side chain were 109 and 119, respectively. In particular, regarding the former, 13 of the 17 severe cases $(76 \%)$ had 40 atoms or more influenced
(Fig. 2, left). On the other hand, the number of influenced atoms in the attenuated MPS I group was low. The averages of the influenced atoms in the main chain and the side chain were 27 and 33, respectively. In particular, regarding main-chain atoms, six of the seven attenuated cases $(88 \%)$ had 39 atoms or less affected (Fig. 2, right). The $F$ test indicated that the distributions exhibited unequal variance $(P<0.05)$ between the two groups, and thus Welch's $t$ test was performed. The results revealed that there was a significant difference in the number of influenced atoms in both the main chain and the side chain $(P<0.05)$ between the severe and attenuated groups. As to the intermediate group, the averages of the influenced atoms in the main chain and the side chain were 65 and 80, respectively, indicating the values are intermediate between those of the severe and attenuated groups. The $F$ test followed by Welch's $t$ test revealed that there were significant differences $(P<0.05)$ in both the main chain and side chain between the severe and milder groups (the intermediate and attenuated groups). However, we could not statistically find any significant differences in the average numbers of atoms influenced between the severe and intermediate groups or between the intermediate and attenuated ones. These results suggest that structural changes caused by the amino acid substitutions responsible for severe MPS I are generally large, those for attenuated MPS I being small, and the structural changes tend to be correlated with the severity of the phenotype.

\section{RMSD for MPS I mutations}

The average RMSDs for the MPS I groups were determined and compared with each other to determine mutation
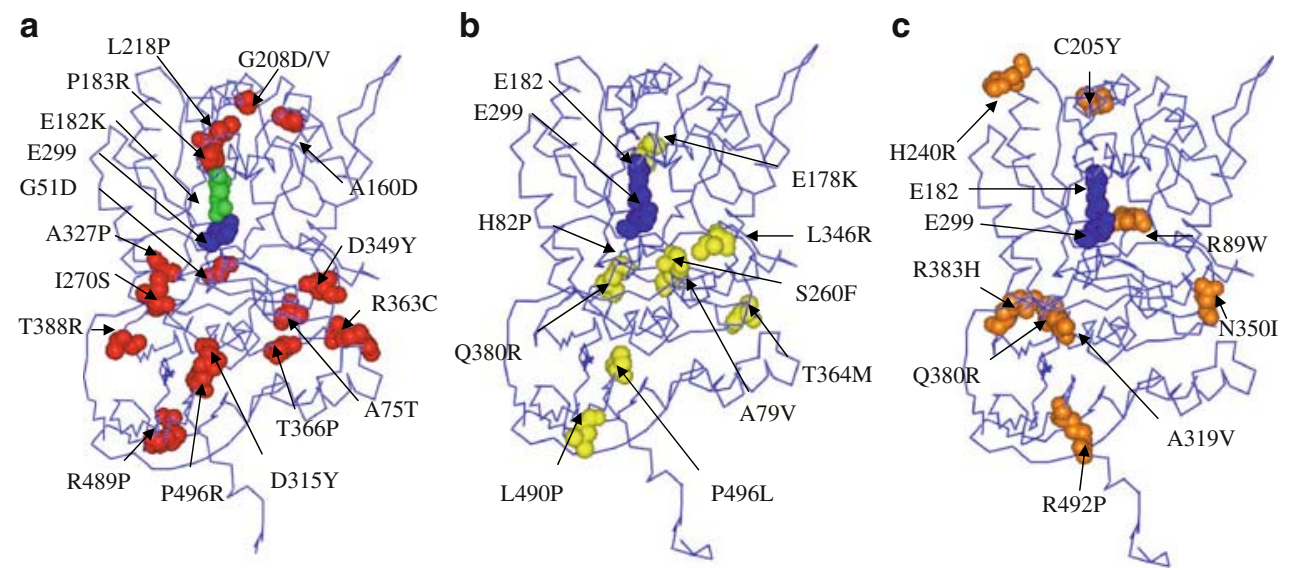

Fig. 1 Localization of missense mutations causing mucopolysaccharidosis type I (MPS I) and amino acid residues involved in the catalysis of the substrate in the three-dimensional structure of $\alpha$-Liduronidases (IDUA). The positions of amino acid substitutions are indicated as space-filling models. $\mathbf{a}, \mathbf{b}$, and $\mathbf{c}$ show the residues for the severe (red), intermediate (yellow), and attenuated (orange) MPS I cases, respectively. The putative acid/base catalyst (E182) and nucleophile (E299) residues in the active site are indicated as space-filling models (blue), except for E182 in a. In a, E182 was indicated as a space-filling model (green), as E182K had been identified in a patient with severe MPS I 

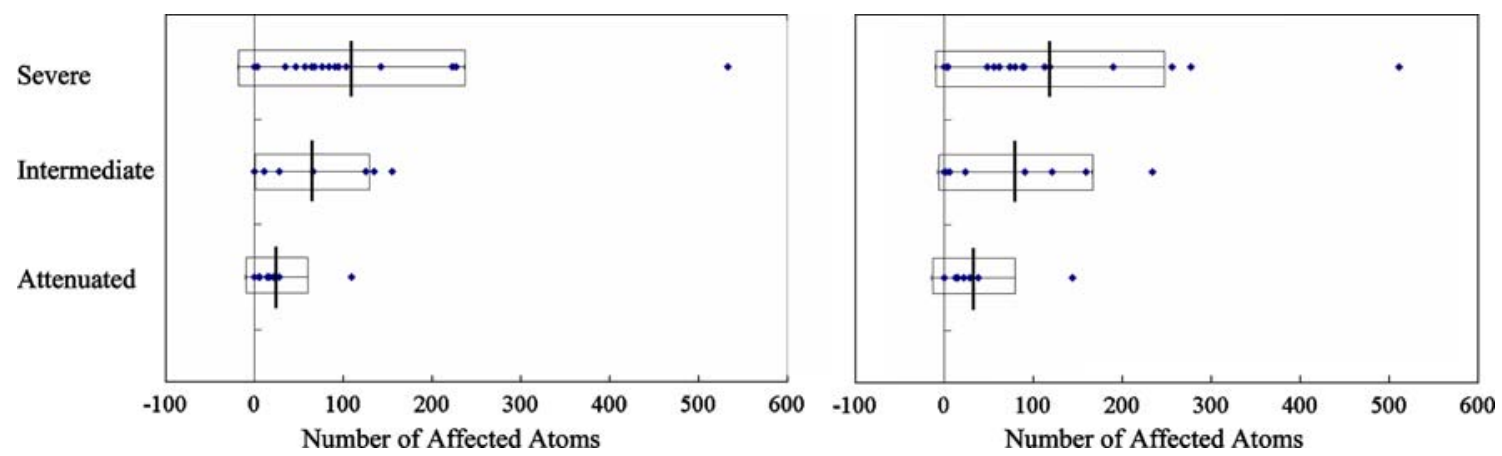

Fig. 2 Numbers of atoms influenced by amino acid substitutions found in severe, intermediate, and attenuated mucopolysaccharidosis type I (MPS I) cases. Main chain (left) and side chain (right). Boxes indicate mean \pm standard deviation

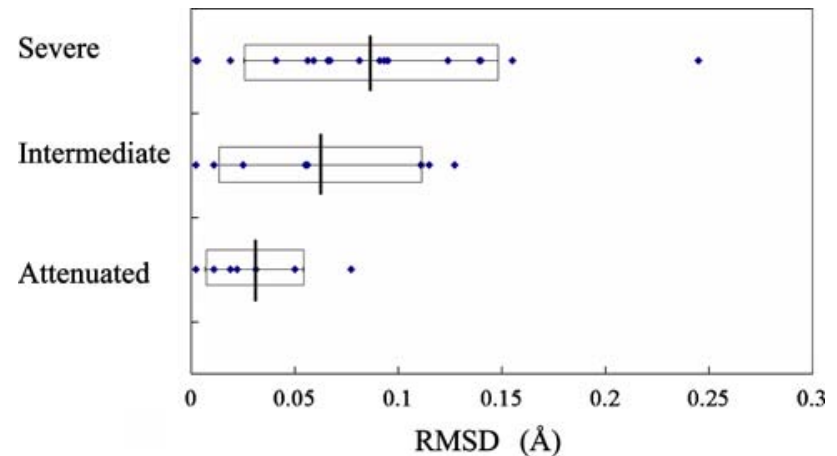

Fig. 3 Root-mean-square distance (RMSD) for severe, intermediate, and attenuated mucopolysaccharidosis type I (MPS I) mutations $(\AA)$. Boxes indicate mean \pm standard deviation

influences. The results are shown in Fig. 3. Those for the severe, intermediate, and attenuated groups were 0.087, 0.063 , and $0.030 \AA$, respectively. Results of the $F$ test and Welch's $t$ test showed that there was a significant difference in RMSD between the severe MPS I and attenuated groups $(P<0.05)$ and between the severe and milder ones (the intermediate and attenuated groups) $(P<0.05)$. No significant difference in the average RMSD between the severe and intermediate groups or between the intermediate and attenuated ones could be found.

ASA of amino acid residues associated with MPS I mutations

To determine locations of the residues associated with MPS I mutations in the structure of IDUA, ASA values of the residues in the wild-type structure were calculated, the results being summarized in Fig. 4 (The result for each residue is shown in the "Supplementary" data). In the severe MPS I group, the ASA range extended to 0.0$60.1 \AA^{2}$, the average being $16.0 \AA^{2}$. In the attenuated group, the ASA range extended to $0.8-146.6 \AA^{2}$, the average being $58.6 \AA^{2}$. The results of the $F$ test $(P<0.05)$ and Welch's $t$ test $(P<0.05)$ showed that there was a

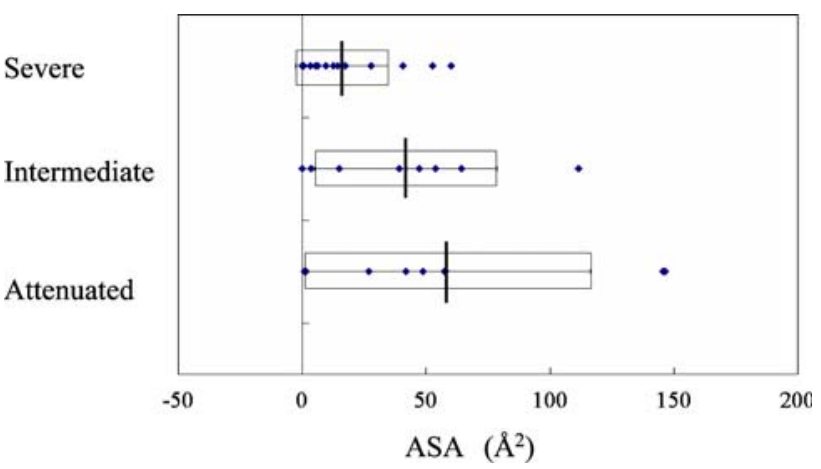

Fig. 4 Solvent-accessible surface area (ASA) of amino acid residues associated with severe, intermediate, and attenuated mucopolysaccharidosis type I (MPS I) $\left(\AA^{2}\right)$. Boxes indicate mean \pm standard deviation

significant difference between the two groups. The average ASA in the intermediate group was $41.8 \AA^{2}$, indicating that it had an intermediate value between those in the severe and attenuated groups. The $F$ test and Welch's $t$ test revealed that there was a significant difference between severe MPS I and milder ones (intermediate and attenuated) $(P<0.05)$. There was also a significant difference in the average ASA between the severe and intermediate ones although not between the intermediate group and the attenuated one. These results indicate that the residues associated with severe MPS I mutations tend to be less solvent-accessible than those associated with the milder MPS I mutations.

Color imaging of structural changes for representative amino acid substitutions causing MPS I

Regarding MPS I mutations, we paid attention to P496R, H240R, and E182K and examined structural changes by means of color imaging. The results are shown in Fig. 5. As shown in Fig. 5a, P496 is buried, and the atoms influenced by P496R are located throughout the whole enzyme. On the other hand, H240 is located on the surface of the 
enzyme protein, and the number of atoms influenced by H240R is limited, as shown in Fig. 5b. Furthermore, color imaging clearly revealed that the active site pocket was severely affected by E182K, as shown in Fig. 5c.

\section{Discussion}

Although a large number of gene mutations causing MPS I have been reported, structural information on a defective IDUA protein is sparse. To elucidate the basis of MPS I, it is very important to examine the structural changes in the enzyme protein responsible for the different phenotypes.

We constructed structural models of MPS I mutant enzyme proteins and examined the correlation between the structural changes and the phenotypes by means of calculation of the numbers of atoms influenced by amino acid substitutions and determination of the RMSD and ASA. Considering the results, the residues associated with severe MPS I mutations tend to be located in the core region of the enzyme protein, and the structural changes are large, which would seriously affect protein folding and/or intracellular transport, which would lead to degradation of the enzyme protein by the endoplasmic reticulum's quality control (ERQC) system before it is transported to the lysosome, as indicated in other lysosomal diseases, including Tay-Sachs disease (Mahuran 1999). Among the mutations responsible for the severe phenotype, the numbers of atoms influenced by A75T, L218P, I270S, and A327P are exceptionally small. However, for L218P and A327P, the ASA values are small, indicating that the substituted residues are buried. The buried residues affected are predicted to cause serious conformation changes, which would affect the stability of the enzyme and result in the severe phenotype.

On the other hand, small structural changes on the surface of the enzyme protein were generally found in the attenuated form. This suggests that at least a small amount of the mutant enzyme can be protected from the ERQC system and transported to the lysosome without losing its activity, and this must lead to residual IDUA activity and thus the late-onset attenuated phenotype. An exceptional case to this "rule" is R89Q. This mutation has been identified in nine of 38 Japanese MPS I alleles (Yamagishi et al. 1996) and also observed in Caucasian Scheie alleles (Scott et al. 1992). The expression study revealed that R89Q was a destabilizing amino acid substitution producing an IDUA protein that had a reduced ability to bind and/ or to turn over a substrate (Scott et al. 1992). However, it remains unclear why the predicted structural change results in residual enzyme activity.

From the results of examination of the intermediate form combined with those for the severe and attenuated groups, the structural changes in the enzyme protein are thought to be correlated with the severity of the phenotype in MPS I.

Color imaging clearly showed the characteristic structural changes caused by the representative amino acid substitutions. P496R causes a large structural change in the core region of the enzyme, which must lead to destabilization and degradation of the expressed protein, resulting in the severe phenotype. H240R is predicted to cause a small structural change on the molecular surface of the protein and thus not to affect the active site. This suggests that at least a small amount of mutant protein having enzyme activity can be protected from the ERQC system and transported to the lysosome, leading to the attenuated phenotype. Color imaging of the atoms in the mutant IDUR with E182K clearly showed a structural change in the active site pocket. Brooks et al. expressed the E182K mutant protein in CHO-K1 cells and found that the expressed protein was enzymatically inactive (Brooks et al. 2001), suggesting that E182K leads to the severe phenotype.

In conclusion, we examined the structural changes in IDUA caused by MPS I mutations. The results show the correlation between the structural changes and clinical phenotypes. Structural investigation is useful for elucidating the basis of MPS I and for increasing our ability to predict the clinical outcome of the disease.

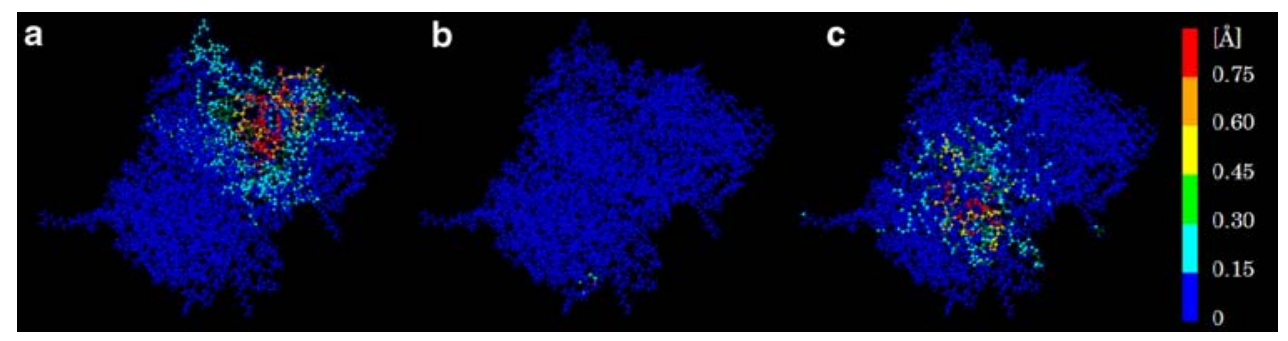

Fig. 5 Color imaging of the atoms in the three-dimensional structure influenced by representative amino acid substitutions causing mucopolysaccharidosis type I (MPS I). The degrees and distributions for P496R (a), H240R (b), and E182K (c) are shown. Each atom is colored according to the distance between the atom in the mutant and the corresponding atom in the wild-type structure. The colors of the atoms show the distances, as follows: blue $<0.15 \AA, 0.15 \AA \leq$ cyan $<0.30 \AA, 0.30 \AA \leq$ green $<0.45 \AA, 0.45 \AA \leq$ yellow $<0.60 \AA$, $0.60 \AA \leq$ orange $<0.75 \AA$, and red $\geq 0.75 \AA$ 
Acknowledgments We thank Dr. J. Ponder (Department of Biochemistry and Molecular Biophysics, Washington University) for providing us with the TINKER software. We also thank I.K. McDonald, D. Naylor, D. Jones, J.M. Thornton, S. Hubbard, D.K. Smith, R. Laskowski, and G. Hutchinson for providing us with the HBPLUS. This work was partly supported by grants from the Japan Society for the Promotion of Science, the Ministry of Education, Science, Sports and Culture of Japan, the Ministry of Health and Welfare of Japan, the Japan Science and Technology Agency, and CREST.

\section{References}

Beesley CE, Meaney CA, Greenland G, Adams V, Vellodi A, Young EP, Winchester BG (2001) Mutational analysis of 85 mucopolysaccharidosis type I families: frequency of known mutations, identification of 17 novel mutations and in vitro expression of missense mutations. Hum Genet 109:503-511

Brooks DA (1993) Review: the immunochemical analysis of enzyme from mucopolysaccharidoses patients. J Inher Metab Dis 16:315

Brooks DA, Fabrega S, Hein LK, Parkinson EJ, Durand P, Yogalingam G, Matte U, Giugliani R, Dasvarma A, Eslahpazire J, Henrissat B, Mornon JP, Hopwood JJ, Lehn P (2001) Glycosidase active site mutations in human $\alpha$-L-iduronidase. Glycobiology 11:741-750

Brooks DA (2002) $\alpha$-L-Iduronidase and enzyme replacement therapy for mucopolysaccharidosis I. Expert Opin Biol Ther 2:967-976

Bunge S, Kleijer WJ, Steglich C, Beck M, Zuther C, Morris CP, Schwinger E, Hopwood JJ, Scott HS, Gal A (1994a) Mucopolysaccharidosis type I: identification of 8 novel mutations and determination of the frequency of the two common alpha-Liduronidase mutations (W402X and Q70X) among European patients. Hum Mol Genet 3:861-866

Bunge S, Steglich C, Kleijer WJ (1994b) Mucopolysaccharidosis type I. Identification of $93 \%$ of mutant alleles in a group of 70 patients. Am J Hum Genet 55:44

Clarke LA, Nelson PV, Warrington CL, Morris CP, Hopwood JJ, Scott HS (1994) Mutation analysis of 19 North American mucopolysaccharidosis type I patients: identification of two additional frequent mutations. Hum Mutat 3:275-282

Dudek MJ, Ponder JW (1995) Accurate modeling of the intramolecular electrostatic energy of proteins. J Comput Chem 16:791816

Kabsch W (1976) A solution for the best rotation to relate two sets of vectors. Acta Crystallogr A32:827

Kabsch W (1978) A discussion of the solution for the best rotation to relate two sets of vectors. Acta Crystallogr A34:922-923

Kong MJ, Ponder JW (1997) Reaction field methods for off-center multipoles. J Chem Phys 107:481-492

Kundrot CE, Ponder JW, Richards FM (1991) Algorithms for calculating excluded volume and its derivative as a function of molecular conformation and their use in energy minimization. J Comput Chem 12:402-409

Laradi S, Tukel T, Erazo M, Shabbeer J, Chkioua L, Khedhiri S, Ferchichi S, Chaabouni M, Miled A, Desnick RJ (2005) Mucopolysaccharidosis I: alpha-L-iduronidase mutations in three Tunisian families. J Inherit Metab Dis 28:1019-1026

Lee-Chen GJ, Wang TR (1997) Mucopolysaccharidosis type I: identification of novel mutations that cause Hurler/Scheie syndrome in Chinese families. J Med Genet 34:939-941

Li P, Wood T, Thompson JN (2002) Diversity of mutations and distribution of single nucleotide polymorphic alleles in the human alpha-L-iduronidase (IDUA) gene. Genet Med 4:420-426
Mahuran DJ (1999) Biochemical consequences of mutations causing the GM2 gangliosidosis. Biochem Biophys Acta 1455:105-108

Matsuzawa F, Aikawa S, Sakuraba H, Lan HT, Tanaka A, Ohono K, Sugimoto Y, Ninomiya H, Doi H (2003) Structural basis of the GM2 gangliosidosis B variant. J Hum Genet 48:582-589

Matsuzawa F, Aikawa S, Doi H, Okumiya T, Sakuraba H (2005) Fabry disease: correlation between structural changes in $\alpha$ galactosidase, and clinical and biochemical phenotype. Hum Genet 117:317-328

Matte U, Yogalingam G, Brooks D, Leistner S, Schwartz I, Lima L, Norato DY, Brum JM, Beesley C, Winchester B, Giugliani R, Hopwood JJ (2003) Identification and characterization of 13 new mutations in mucopolysaccharidosis type I patients. Mol Genet Metab 78:37-43

McDonald IK, Thornton JM (1994) Satisfying hydrogen bonding potential in proteins. J Mol Biol 238:777-793

Neufeld EF, Muenzer J (2001) The mucopolysaccharidoses. In: Scriver CR, Beaudet AL, Sly WS, Valle D (eds) The metabolic and molecular bases of inherited disease, 8th edn. McGraw-Hill, New York, pp 3421-3452

Pappu RV, Hart RW, Ponder JW (1998) Analysis and application of potential energy smoothing for global optimization. J Phys Chem B102:9725-9742

Rempel BP, Clarke LA, Withers SG (2005) A homology model for human $\alpha$-L-iduronidase: insights into human disease. Mol Genet Metab 85:28-37

Ren P, Ponder JW (2003) Polarizable atomic multipole water model for molecular mechanics simulation. J Phy Chem B107:5933-5947

Sakuraba H, Matsuzawa F, Aikawa S, Doi H, Kotani M, Lin H, Ohno K, Tanaka A, Yamada H, Uyama E (2000) Molecular and structural studies of the GM2 gangliosidosis $\mathrm{O}$ variant. J Hum Genet 47:176-183

Sakuraba H, Matsuzawa F, Aikawa S, Doi H, Kotani M, Nakada H, Fukushige T, Kanzaki T (2004) Structural and immunocytochemical studies on $\alpha-N$-acetylgalactosaminidase deficiency (Schindler/Kanzaki disease). J Hum Genet 49:1-8

Scott HS, Ashton LJ, Eyre HJ, Backer E, Brooks DA, Callen DF, Sutherland GR, Morris CO, Hopwood JJ (1990) Chromosomal localization of the human $\alpha$-L-iduronidase (IDUA) to $4 \mathrm{p} 16.3$. Am J Hum Genet 47:802-807

Scott HS, Guo X-H, Hopwood JJ, Morris CP (1992) Structure and sequence of the $\alpha$-L-iduronidase gene. Genomics 13:1311-1313

Scott HS, Litjens T, Nelson PV, Thompson PR, Brooks DA, Hopwood JJ, Morris CP (1993) Identification of mutations in the alpha-L-iduronidase gene (IDUA) that cause Hurler and Scheie syndromes. Am J Hum Genet 53:973-986

Scott HS, Bunge S, Gal A, Clarke LA, Morris CP, Hopwood JJ (1995) Molecular genetics of mucopolysaccharidosis type I: diagnostic, clinical, and biological implications. Hum Mutat 6:288-302

Teng YN, Wang TR, Hwu WL, Lin SP, Lee-Chen GJ (2000) Identification and characterization of $-3 \mathrm{c}-\mathrm{g}$ acceptor splice site mutation in human alpha-L-iduronidase associated with mucopolysaccharidosis type IH/S. Clin Genet 57:131-136

Tieu PT, Bach G, Matynia A, Hwang M, Neufeld EF (1995) Four novel mutations underlying mild or intermediate forms of alphaL-iduronidase deficiency (MPS IS and MPS IH/S). Hum Mutat 6:55-59

Tylor JA, Gibson GJ, Brooks DA, Hopwood JJ (1991) $\alpha$-L-iduronidase in normal and mucopolysaccharidosis type I human skin fibroblasts. Biochem J 274:263-268

Venturi N, Rovelli A, Parini R, Menni F, Brambillasca F, Bertagnolio F, Uziel G, Gatti R, Filocamo M, Donati MA, Biondi A, Goldwurm S (2002) Molecular analysis of 30 mucopolysaccharidosis type I patients: evaluation of the mutational spectrum in Italian population and identification of 13 novel mutations. Hum Mutat 20:231 
Weiner SJ, Kallman PA, Case DA, Singh UC, Ghio C, Alagona G, Profeta S, Weiner P (1984) A new force field for molecular mechanical simulation of nucleic acids and proteins. J Am Chem Soc 106:765-784

Yamagishi A, Tomatsu S, Fukuda S, Uchiyama A, Shimozawa N, Suzuki Y, Kondo N, Sukegawa K, Orii T (1996) Mucopolysaccharidosis type I: identification of common mutations that cause
Hurler and Scheie syndrome in Japanese populations. Hum Mut 7:23-29

Yogalingam G, Guo XH, Muller VJ, Brooks DA, Clements PR, Kakkis ED, Hopwood JJ (2004) Identification and molecular characterization of alpha-L-iduronidase mutations present in mucopolysaccharidosis type I patients undergoing enzyme replacement therapy. Hum Mutat 24:199-207 